\title{
THE EFFECTS OF OUTLIERS ON THE TEST FOR SYMMETRY IN PRICE TRANSMISSION MODELS
}

\author{
Acquah De-Graft H., Associate Professor \\ Department of Agricultural Economics and Extension, University of Cape Coast, \\ Cape Coast, Ghana \\ E-mail: henrydegraftacquah@yahoo.com
}

\begin{abstract}
Monte Carlo experimentation is conducted to investigate the effects of outlier observations on the test of symmetry in the Houck and Granger and Lee asymmetric price transmission data generating process. The results of the Monte Carlo experimentation indicate that fewer outliers show little modification in the probability of Type I error rates for the test of symmetry in the Houck and Granger and Lee models. However, rejection frequencies of the true null hypothesis of symmetry rises with increase in the number of outliers in the data generating process. The simulation results indicate that the severity of outlier effects on the test of symmetry depends on sample size and the underlying asymmetric price transmission data generating process. With large amount of outliers and large sample size, the Houck's model detects more spurious asymmetries in the symmetric data generating process than the Granger and Lee model. These results confirm the claims of Douglas (2010) that outliers in the price data could generate asymmetric price response.
\end{abstract}

\section{KEY WORDS}

Outliers, Monte Carlo simulations, power, asymmetric price transmission models, type I error.

An important area of research within price transmission analysis has focused on investigating asymmetries in the price transmission process. Subsequently, numerous studies (Douglas (2010), Meyer and von Cramon-Taubadel (2004), Abdulai (2002), Peltzman (2000), von Cramon-Taubadel (1998), Borenstein et al. (1997), Hahn (1990), Bailey and Brorsen (1989), Kinnucan and Forker (1987) and Ward (1982) find evidence of price asymmetry in agricultural and related markets. However, the evidence of price asymmetry or non-linearity could result from the presence of outliers in data. Kind (2015) notes that price data used in price transmission analysis contains outliers. This is worrying since measuring asymmetry with data containing outliers could lead to misleading inference on price asymmetry and policy implications. The importance of outliers in asymmetric price transmission analysis is noted in previous research using empirical price data. For example, Douglas (2010) claims that asymmetry found is caused by outliers in data. He notes that asymmetry disappears after exclusion of outliers in the data.

The effect of outliers on hypothesis testing has been mentioned several times in the economics and statistics literature. In the economics literature some studies (Ahmad \& Glosser 2011; Koop \& Potter, 2001; Van Dijk, Franses \& Lucas, 1999; Ahmed, 2008; Lopez Villavicencio, 2008; Ahamed \& Donayre, 2002; and Tolvi, 2000) examined the effect of outliers on the test for non-linearity. These studies found that non-linearity is reflected in a small number of outlier observations in the data that they examined. They noted that outlier observations can distort linear relationship and cause the linearity test to reject the correct null hypothesis of linearity too often. However, these studies did not investigated the effect of outliers on commonly used asymmetric price transmission models (Houck and Granger and Lee models). In the statistics literature, some studies (Liao, Li \& Brooks, 2016; Barnett \& Lewis, 1994; Hampel, Ronchetti, Rousseeuw, \& Stahel, 1986; Wilcox, 1998; and Zimmerman, 1994b) have provided evidence that shows the effect of outliers resulted in inflation of Type I error rates and reduced power in parametric $t$ and $F$ tests.

Aside Douglas (2010) claims that outliers cause price asymmetry, little is known about the effect of outliers on the test for non-linearity in asymmetric price transmission analysis. 
Empirically, less effort has been made in examining the effect of outliers on the test for nonlinearity within the asymmetric price transmission modelling context. Notably, the ability of the commonly used asymmetric price transmission models to cause the linearity test to reject the correct null hypothesis of linearity too often in the presence of outliers have not yet been extensively investigated and is not well understood. An important question which remains unanswered is how well will the test for non-linearity perform in the Houck and Granger and Lee models when outliers are present in the data used for price transmission analysis. In the presence of outliers, will there be inflation of Type I error rates and reduced power in the test for non-linearity in asymmetric price transmission models? And will the severity of outlier effects on the test of symmetry depend on sample size and the underlying asymmetric price transmission data generating process?

In order to address this issue, this paper supports Douglas (2010)'s claim that outliers in price data causes asymmetry and empirically evaluate the effect of outliers on the test for symmetry in two commonly used asymmetric price transmission models ( Houck and Granger and Lee models) via Monte Carlo experimentation. The paper contributes towards understanding the effect of outliers on the test for symmetry in Houck and Granger and Lee Models. The true data generating process is known in all experiments and the Monte Carlo simulations are essential in deriving the Type I error rates. Specifically, using simulated data, it is shown that the presence of outliers in a symmetric price data generating process lead to spurious asymmetries and could make the symmetry test reject the true null hypothesis of symmetry.

First, the study evaluates the power of the test for symmetry in the symmetric Houck's and Granger and Lee data generating process when outliers are absent. Second, the effect of outliers on the test for symmetry is investigated by introducing outliers into the symmetric Houck's and Granger and Lee data generating process. This study differs from existing literature in that it is the only one that explores the effect of outliers on the Houck and Granger and Lee asymmetric price transmission data generating process in a Monte Carlo experimentation.

\section{METHODS OF RESEARCH}

Measuring Asymmetry. Wolffram (1971) proposed an approach to measuring asymmetric price transmission that draws from Tweenten and Quance (1969). Houck (1977) suggested a simple but operationally clear alternative to the Wolffram approach to measuring asymmetric price transmission. However, these approaches do not take into consideration the long-run equilibrium relationship between the variables. Von Cramon Taubadel (1998) provides an alternative approach to measuring asymmetry that takes into consideration the concept of cointegration which considers the long- run equilibrium relationship between the variables. This model draws from Granger and Lee (1989).

Measuring Asymmetry using the Houck's Model. Houck (1977) suggested a simple model for testing for asymmetry as follows:

$$
\Delta \mathrm{y}_{t}=\beta_{1}^{+} \Delta x_{t}^{+}+\beta_{1}^{-} \Delta x_{t}^{-}+\varepsilon \quad \varepsilon \sim N\left(0, \sigma_{\varepsilon}^{2}\right)
$$

Where: $\Delta x_{t}^{+}$and $\Delta x_{t}^{-}$are the positive and negative changes in $x_{t}$. The explanatory variable $x_{t}$ is generated as independent draws from normal distribution with a constant mean and a variance of one. In order to introduce asymmetry into the transmission process, speeds of adjustments for the coefficients of $\Delta x_{t}^{+}$and $\Delta x_{t}^{-}$in eqn. (1) are allowed to differ and the errors $(\varepsilon)$ are generated as independently and identically distributed draws from the standard normal distribution with a sample size $n$. $\Delta \mathrm{y}_{t}$ can be constructed using the values for beta, positive and negative changes in $x_{t}$ (i.e. $\Delta x_{t}^{+}$and $\Delta x_{t}^{-}$) and the error term as 
specified in eqn. (1). Symmetric price transmission behavior is tested by determining whether the coefficients ( $\beta_{1}^{+}$and $\beta_{1}^{-}$) are identical (i.e. $H_{0}: \beta_{1}^{+}=\beta_{1}^{-}$).

Measuring Asymmetry using Granger and Lee Model. Consider the following simple Granger and Lee (1989) asymmetric error correction model data generating process which shows the adjustment in variable $y$ in response to deviations from its equilibrium relationship with variable $\mathrm{x}$. Notably, $y_{t}$ and $x_{t}$ are generated as non-stationary variables that are integrated of the order one. A cointegrating relationship exist between $y$ and $x$ which is defined by the error correction term. The positive and negative components of the error correction term are denoted by $\left(y_{t}-x_{t}\right)^{+}{ }_{t-1}$ and $\left(y_{t}-x_{t}\right)^{-}{ }_{t-1}$.

The errors $\varepsilon$ are normally distributed with a mean of zero and a variance of one.

$$
\Delta y_{t}=\theta_{1} \Delta x_{t}-\theta_{2}^{+}(y-x)_{t-1}^{+}-\theta_{2}^{-}(y-x)_{t-1}^{-}+\varepsilon_{t}
$$

To allow for asymmetric adjustments, the equilibrium relationship is partitioned as follows:

$$
\begin{aligned}
& (y-x)_{t-1}^{+}= \begin{cases}(y-x)_{t-1} \text { if }(y-x)_{t-1}>0 \\
0 & \text { Otherwise }\end{cases} \\
& (y-x)_{t-1}^{-}= \begin{cases}(y-x)_{t-1} & \text { if }(y-x)_{t-1}<0 \\
0 & \text { Otherwise }\end{cases}
\end{aligned}
$$

Symmetry behavior in eqn. (2) is detected by determining whether the coefficients $\left(\theta_{2}^{+}\right.$ and $\theta_{2}^{-}$) are identical (that is $H_{0}: \theta_{2}^{+}=\theta_{2}^{-}$).

\section{RESULTS AND DISCUSSION}

In order to investigate the effect of outliers on the test for asymmetry under various sample size conditions, a series of Monte Carlo simulations of the Houck and Granger and Lee models are carried out based on 10000 replications. In particular, effect of various percentages of outliers on the test for non-linearity in two asymmetric price transmission models (Houck and Granger and Lee models) are investigated under conditions of different sample sizes when the true data generating process is symmetric with the levels of asymmetry given by:

$$
\left(\beta_{2}^{+}, \beta_{2}^{-}\right)=\left(\theta_{2}^{+}, \theta_{2}^{-}\right) \in(0.25,0.25)
$$

The Houck's symmetric data generating process is specified as follows:

$$
\Delta \mathrm{y}_{t}=0.25 \Delta x_{t}^{+}+0.25 \Delta x_{t}^{-}+\varepsilon
$$

The Granger and Lee symmetric data generating process is specified as follows:

$$
\Delta y_{t}=0.50 \Delta x_{t}-0.25(y-x)_{t-1}^{+}-0.25(y-x)_{t-1}^{-}+\varepsilon
$$

The assignment of asymmetric adjustment parameters draws from Holly et. al., (2003). The errors are generated from a normal distribution with a mean 0 and a variance of 1 for data without outliers. Data with outliers is created by introducing various percentages of outliers (0-5 percents) into the data. For example, one percent of the number of observations 
of the errors generated for the normal data with values generated from a normal distribution with a mean of 0 and a variance of 1 , were replaced with one percent of the number of observations from the normal distribution with a mean of 20 and variance of 1 for a chosen sample size. This is repeated for 2, 3, 4 and 5 percent of outliers given the various sample sizes 150,300 and 500 respectively. The data generating processes are simulated 10000 times with different percentages of outliers and across different sample sizes. The performance of the symmetry test of the Houck and Granger and Lee models are evaluated in terms of their ability to reject the true null hypothesis of symmetric adjustment using an Ftest of the restricted versus the unrestricted model when the data has no outliers or is contaminated with outliers. The results in Table 1 indicate the rejection frequencies of the true null hypothesis of symmetry. For an example, a rejection frequency of 0.0506 in Table 1 for 0 outliers and a sample size of 150 imply that the null hypothesis of symmetry is rejected $5.06 \%$ of the time for the Granger and Lee model.

Table 1 - Rejection Frequencies for Symmetry Test - Granger and Lee Model

\begin{tabular}{|c|c|c|c|c|c|c|}
\hline \multirow{2}{*}{ Outlier \% } & \multicolumn{6}{|c|}{ Sample Size(N) and 5\% Rejection Frequency } \\
\cline { 2 - 6 } & $\mathrm{N}$ & Rej (5\%) & $\mathrm{N}$ & $\operatorname{Rej}(5 \%)$ & $\mathrm{N}$ & Rej (5\%) \\
\hline 0 & 150 & 0.0506 & 300 & 0.0540 & 500 & 0.0518 \\
\hline 1 & 150 & 0.0501 & 300 & 0.0500 & 500 & 0.0500 \\
\hline 2 & 150 & 0.0528 & 300 & 0.0970 & 500 & 0.3827 \\
\hline 3 & 150 & 0.0537 & 300 & 0.3203 & 500 & 0.8003 \\
\hline 4 & 150 & 0.0909 & 300 & 0.6128 & 500 & 0.9655 \\
\hline 5 & 150 & 0.2510 & 300 & 0.9613 & 500 & 0.9976 \\
\hline
\end{tabular}

Based on 10,000 replications.

The study examined the rejection frequencies of the true null hypothesis of symmetry when there are no outliers in the simulated data. Notably, the study finds asymmetry in its absence. The results show that the Type I error rates of the F-test was around the significance level of 0.05 when the data has no outliers as illustrated in upper part of Tables 1 and 2. Similarly, Liao, Li and Brooks (2016) found that when outliers are removed from the dataset, the Type I error rates of ANOVA tests drop back to a significance level of around 0.05 .

Table 2 - Rejection Frequencies for Symmetry Test - Houck's Model

\begin{tabular}{|c|c|c|c|c|c|c|}
\hline \multirow{2}{*}{ Outlier \% } & \multicolumn{7}{|c|}{ Sample Size (N) and 5\% Rejection Frequency } \\
\cline { 2 - 6 } & $\mathrm{N}$ & Rej (5\%) & $\mathrm{N}$ & Rej (5\%) & $\mathrm{N}$ & Rej (5\%) \\
\hline 0 & 150 & 0.0506 & 300 & 0.0500 & 500 & 0.0500 \\
\hline 1 & 150 & 0.0501 & 300 & 0.0515 & 500 & 0.0500 \\
\hline 2 & 150 & 0.0509 & 300 & 0.0999 & 500 & 0.3830 \\
\hline 3 & 150 & 0.0527 & 300 & 0.3230 & 500 & 1.0000 \\
\hline 4 & 150 & 0.0950 & 300 & 0.6024 & 500 & 1.0000 \\
\hline 5 & 150 & 0.2375 & 300 & 0.9629 & 500 & 1.0000 \\
\hline
\end{tabular}

Based on 10,000 replications.

The study also examined the rejection frequencies of the true null hypothesis of symmetry when the data is contaminated with outliers. The study found a clear effect of outliers on the test for non-linearity. Fewer outliers show little modification in the probability of Type I error rates for the test of symmetry. This becomes evident when rejection frequencies obtained for data with 0 and 1 percent outlier contamination are compared under the Houck and Granger and Lee models in Tables 1 and 2. Similarly, Liao, Li and Brooks (2016) note that fewer outliers show little modification in the probability of Type I error rates. However, increasing the number of outliers in the various samples increased the probability of the Type I error for the test of symmetry. Increasing the outlier percentage from $2 \%$ to $5 \%$ increased the rejection frequencies of the true null hypothesis of symmetry for different sample sizes. For example, in Table 1, as outlier percentages increase from $2 \%$ to $5 \%$, rejection 
frequencies of the true null hypothesis of symmetry in the Granger and Lee model increased from $5.28 \%$ to $25.10 \%, 9.70 \%$ to $96.13 \%$ and $38.27 \%$ to $99.76 \%$ for sample sizes of 150 , 300 and 500 respectively at 5\% significance levels. Similarly, in Table 2 as outlier percentages increase from $2 \%$ to $5 \%$, rejection frequencies of the true null hypothesis of symmetry in the Houck's model increased from $5.09 \%$ to $23.75 \%, 9.99 \%$ to $96.29 \%$ and $38.30 \%$ to $100 \%$ for sample sizes of 150,300 and 500 respectively at $5 \%$ significance level.

Table 3 - Rejection Frequencies for Symmetry Test - Granger and Lee Model

\begin{tabular}{|c|c|c|c|c|c|c|}
\hline \multirow{2}{*}{ Outlier \% } & \multicolumn{7}{|c|}{ Sample Size(N) and 1\% Rejection Frequency } \\
\cline { 2 - 6 } & $\mathrm{N}$ & Rej (1\%) & $\mathrm{N}$ & Rej (1\%) & $\mathrm{N}$ & Rej (1\%) \\
\hline 0 & 150 & 0.0101 & 300 & 0.0115 & 500 & 0.0113 \\
\hline 1 & 150 & 0.0117 & 300 & 0.0108 & 500 & 0.0103 \\
\hline 2 & 150 & 0.0126 & 300 & 0.0206 & 500 & 0.1291 \\
\hline 3 & 150 & 0.0144 & 300 & 0.0957 & 500 & 0.4559 \\
\hline 4 & 150 & 0.0198 & 300 & 0.2558 & 500 & 0.7815 \\
\hline 5 & 150 & 0.0632 & 300 & 0.7563 & 500 & 0.9467 \\
\hline
\end{tabular}

Based on 10,000 replications.

Table 4 - Rejection Frequencies for Symmetry Test - Houck's Model

\begin{tabular}{|c|c|c|c|c|c|c|}
\hline \multirow{2}{*}{ Outlier \% } & \multicolumn{6}{|c|}{ Sample Size $(\mathrm{N})$ and 1\% Rejection Frequency } \\
\hline & $\mathrm{N}$ & $\operatorname{Rej}(1 \%)$ & $\mathrm{N}$ & $\operatorname{Rej}(1 \%)$ & $\mathrm{N}$ & $\operatorname{Rej}(1 \%)$ \\
\hline 0 & 150 & 0.0101 & 300 & 0.0109 & 500 & 0.0109 \\
\hline 1 & 150 & 0.0110 & 300 & 0.0112 & 500 & 0.0104 \\
\hline 2 & 150 & 0.0103 & 300 & 0.0236 & 500 & 0.1270 \\
\hline 3 & 150 & 0.0123 & 300 & 0.0958 & 500 & 0.9970 \\
\hline 4 & 150 & 0.0190 & 300 & 0.2605 & 500 & 0.9996 \\
\hline 5 & 150 & 0.0603 & 300 & 0.7630 & 500 & 1.0000 \\
\hline
\end{tabular}

Based on 10,000 replications.

Similar patterns are observed for the Houck and Granger and Lee models at $1 \%$ significance levels in Table 3 and 4. Notably, as outlier percentages increase from $2 \%$ to $5 \%$, rejection frequencies of the true null hypothesis of symmetry in the Granger and Lee model increased from $1.26 \%$ to $6.32 \%, 2.06 \%$ to $75.63 \%$ and $12.91 \%$ to $94.67 \%$ for sample sizes of 150,300 and 500 respectively at $1 \%$ significance level in Table 3. Similarly, in Table 4 as outlier percentages increase from $2 \%$ to $5 \%$, rejection frequencies of the true null hypothesis of symmetry in the Houck's model increased from $1.03 \%$ to $6.03 \%, 2.36 \%$ to $76.30 \%$ and $12.70 \%$ to $100 \%$ for sample sizes of 150,300 and 500 respectively at $1 \%$ significance level. In effect rejection frequencies of the true null hypothesis of symmetry rises as the amount of outliers increase in individual samples. Similarly, some studies (Barnett \& Lewis, 1994; Hampel, Ronchetti, Rousseeuw, \& Stahel, 1986; Wilcox, 1998; Zimmerman, 1994b) have provided evidence that shows the effect of outliers resulted in inflation of Type I error rates and reduced power in parametric t and F tests. Similarly, Liao, Li and Brooks (2016) also found that with an increasing number of outliers being injected into the sample, the probability of the Type I error rates of the ANOVA test increased substantially.

Other factors which influences the rejection frequencies of the true null hypothesis of symmetry (Type I error rates) are the sample size and underlying asymmetric data generating process. At $5 \%$ outlier contamination, there is some increase in rejection frequencies when the sample size is increased 150 to 500 for both the Houck and the Granger and Lee models. At $5 \%$ outlier contamination, rejection frequencies for the true null hypothesis of symmetry in the Granger and Lee model are $25.10 \%, 96.13 \%$ and $99.76 \%$ for sample sizes of 150,300 and 500 respectively at 5\% significance level. Similarly, at $5 \%$ outlier contamination, rejection frequencies for the null hypothesis of symmetry in the Houck's model are $23.75 \%, 96.29 \%$ and $100 \%$ for sample sizes of 150,300 and 500 respectively at $5 \%$ significance level. These results suggest that as sample size increases, the Type I error rates increases but at a decreasing rate. Using simulated populations, Liao, $\mathrm{Li}$ and Brooks (2016) noted that the magnitude of Type I error rate inflation decreases with 
the growth of sample size. In other words, the impact of outliers on the false rejection rates is substantially greater with smaller sample sizes, and as sample size increases, the impact of outliers decreases although it is still inflated. With large amount of outliers (5\%) and large sample size, the Houck's model detect more spurious asymmetries in the symmetric data generating process than the Granger and Lee model. These results indicate that the severity of outlier effects on the test of symmetry depends on sample size as well as the underlying asymmetric data generating process.

\section{CONCLUSION}

The study examined the effect of outliers on the test for symmetry in the Houck and Granger and Lee asymmetric price transmission models. Generally, the Monte Carlo simulation results indicate that fewer outliers show little change in the probability of Type I error rates for the test of symmetry in the Houck and Granger and Lee models. However, increasing the amount of outliers increase the ability of the symmetry test to reject the correct null hypothesis of symmetry in the asymmetric price transmission models studied. In summary, the presence of outliers lead to spurious asymmetries and could make the symmetry test reject the correct null hypothesis of symmetry. These results confirm the claims of Douglas (2010) that outliers in the price data could generate price asymmetry. With large amount of outliers (5\%) and large sample size, the Houck's model detect more spurious asymmetries in the symmetric data generating process than the Granger and Lee model. In effect, simulation results indicate that the severity of outlier effects on the test of symmetry depends on sample size and the underlying asymmetric price transmission data generating process.

The study contributes to knowledge and understanding of the effects of outliers on the test for symmetry in asymmetric price transmission modelling framework. Additionally, the study contributes to the literature on asymmetric price transmission modelling by making researchers aware of the tendency of the test for symmetry to reject correct null hypothesis of symmetry in the asymmetric price transmission models when outlier percentages in data are high.

\section{REFERENCES}

1. Abdulai, A. (2002). Using Threshold Cointegration to Estimate Asymmetric Price Transmission in the Swiss Pork Market. Applied Economics, 34, pp. 679-687

2. Ahmad, Y. S. \& Glosser, S. (2011), "Searching for Nonlinearities in Real Exchange Rates," Applied Economics, 43, 1829-1845

3. Ahmad, Y. S. (2008), "The Effects of Small Sample Bias in Threshold Autoregressive Models" Economics Letters, 101, 6-8

4. Ahmad, Y \& Donayre L. (2002). Outliers and Persistence in Threshold Autoregressive Processes: A Puzzle? Working Paper, University of Wisconsin-Whitewater

5. Bailey, D. \& Brorsen, B.W. (1989). Price Asymmetry in Spatial Fed Cattle Market, Western Journal of Agricultural Economics, Vol. 14 (2), pp. 246-252

6. Barnett, V. \& Lewis, T. (1994). Outliers in Statistical Data (3rd ed.) Chichester, UK: Wiley

7. Borenstein, S., Cameron, A. C. \& Gilbert, R. (1997). DO Gasoline prices respond asymmetrically to Crude Oil Price Changes?, Quarterly Journal of Economics, 112, pp. 305- 339.

8. Douglas, C. C. (2010). Do Gasoline Prices Exhibit Asymmetry? Not usually!. Energy Economics, 32(4), 918-925

9. Granger, C.W. J., \& Lee, T.H. (1989). Investigation of Production, Sales and Inventory Relationships using Multicointegration and non-symmetric Error Correction Models. Journal of Applied Econometrics 4, pp. 135- 159

10. Hahn, W. F. (1990). Price Transmission Asymmetry in Pork and Beef Markets. The Journal of Agricultural Economics Research, 42, No. 4, pp. 21-30 
11. Hampel, F. R., Ronchetti, E. M., Rousseeuw, P.J., \& Stahel, W. A. (1986). Robust statistics: The approach based on influence functions. New York, NY: Wiley

12. Holly, S., Turner, P. \& Weeks, M. (2003). Asymmetric Adjustment and Bias in Estimation of an Equilibrium Relationship from a Cointegrating Regression. Computational Economics, 21, pp. 195-202

13. Houck, J. P. (1977). An Approach to Specifying and Estimating Nonreversible Functions, American Journal of Agricultural Economics, 59, pp. 570-572

14. Kind, M (2015). Analysis of Market Integration- An Alternative Approach. MSc Thesis submitted to the Agricultural Economics and Rural Policy Group, Wageningen University, Netherland

15. Kinnucan, H.W. \& Forker, O. D. (1987). Asymmetry in Farm-Retail Price Transmission for major Dairy Products", American Journal of Agricultural Economics, 69, pp. 285-292

16. Koop, G. \& Potter, S. (2001), "Are Apparent Findings of Nonlinearity due to Structural Instability in Economic Time Series?" Econometrics Journal, 4, 37-55

17. Liao, H., Li, Y. \& Brooks, G. (2016). Outlier Impact and Accommodation Methods: Multiple Comparisons of Type 1 Error Rates, Journal of Modern Applied Statistical Methods, volume 15

18. Lopez Villavicencio, A. (2008), "Nonlinearities or Outliers in Real Exchange Rates?" Economic Modelling, 25, 714-730

19. Meyer, J. \& von Cramon-Taubadel, S. (2004): Asymmetric Price Transmission: A survey. Journal of Agricultural Economics, 55 (3), pp. 581-611

20. Peltzman, S. (2000). Prices Rise Faster than They Fall. Journal of Political Economy, 108, No. 3, pp. 466-502

21. Tolvi, J. (2000). The Effects of Outliers on Two Nonlinearity Tests, Communications in Statistics-Simulation and Computation, 29:3, 897-918

22. Tweenten, L.G. \& Quance, C. L. (1969). Positivistic Measures of aggregate Supply Elasticities: Some new Approaches. American Journal of Agricultural Economics, 51, pp. 342-352

23. Van Dijk, D., Franses, P. H. \& Lucas, A. (1999), "Testing for Smooth Transition Nonlinearity in the Presence of Outliers", Journal of Business and Economics Statistics, $17(2), 217-235$

24. Von Cramon-Taubadel, S. (1998). Estimating Asymmetric Price Transmission with the Error Correction Representation: An Application to the German Pork Market", European Review of Agricultural Economics, 25, pp. 1-18

25. Ward, R. W. (1982). Asymmetry in Retail, Wholesale and Shipping Point Pricing for fresh Vegetables. American Journal of Agricultural Economics, 62, pp. 205-212

26. Wilcox, R. R. (1998). How many discoveries have been lost by ignoring modern statistical methods? American Psychologist, 53(3), 300-314. doi: 10.1037/003-066X. 53.3.300

27. Wolffram, R. (1971). "Positivistic Measures of Aggregate Supply Elasticities-Some New Approaches -Some Critical Notes," American journal of Agricultural Economics, 53, pp. 356-356

28. Zimmerman, D. W. (1994b). A Note on the Influence of Outliers on Parametric and Nonparametric Tests. The journal of General Psychology, 121(4),391-401. doi: 10.1080/00221309.1994.9921213 